\title{
On the Theory of Business-Cycle Control
}

\section{J. Tinbergen}

Econometrica, Vol. 6, No. 1. (Jan., 1938), pp. 22-39.

Stable URL:

http://links.jstor.org/sici?sici=0012-9682\%28193801\%296\%3A1\%3C22\%3AOTTOBC\%3E2.0.CO\%3B2-K

Econometrica is currently published by The Econometric Society.

Your use of the JSTOR archive indicates your acceptance of JSTOR's Terms and Conditions of Use, available at

http://www.jstor.org/about/terms.html. JSTOR's Terms and Conditions of Use provides, in part, that unless you have obtained prior permission, you may not download an entire issue of a journal or multiple copies of articles, and you may use content in the JSTOR archive only for your personal, non-commercial use.

Please contact the publisher regarding any further use of this work. Publisher contact information may be obtained at http://www.jstor.org/journals/econosoc.html.

Each copy of any part of a JSTOR transmission must contain the same copyright notice that appears on the screen or printed page of such transmission.

JSTOR is an independent not-for-profit organization dedicated to and preserving a digital archive of scholarly journals. For more information regarding JSTOR, please contact support@jstor.org. 


\section{ON THE THEORY OF BUSINESS-CYCLE CONTROL}

\section{By J. TiNBERgen}

\section{INTRODUCTION}

The PRESENT STUDY consists of two kinds of approach: (1) a statistical research into the chief dynamic equations describing American business cycles between 1920 and 1932 and (2) a number of more general methodological remarks on this type of research with particular attention to problems of policy. In order to simplify exposition in many points, it seemed desirable to exemplify the general thought immediately; and for this reason the two approaches are more or less mixed.

\section{A MODEL OF AMERICAN ECONOMY FOR 1919-1932}

In order to fix the ideas and to have a possibility of application and exemplification, a very simple model of American business life may be introduced. The variables are:

$$
\begin{aligned}
Z & =\text { Total nonlabour income earned, } \\
F^{\prime} & =\text { Expenditures by nonworkers for consumers' goods, } \\
B & =\text { Employment in investment industries. }
\end{aligned}
$$

$Z$ and $F^{\prime}$ are measured in such units as to make the average value of $Z$ over the period 1919-1932 equal to one; the units of $B$ are such that the average value of total employment over 1919-1932 equals one. The unit of time is taken equal to 4 months.

The choice of the variables and their units is less arbitrary than would seem at first sight. The model is generated, by small simplifications, from a more complicated one that would seem more logical. This has been described elsewhere. ${ }^{1}$ The figures used for the variables and their computation are indicated in Table 1.

The equations are of the following type:

1. An institutional equation indicating the calculation of nonlabour income:

$$
Z_{t}=F_{t}{ }^{\prime}+B_{t}+\zeta_{B} B_{t-1}+A^{0}+A^{1} t+\zeta_{Z}\left(Z_{t-1}-Z_{t-2}\right)
$$

2. A reaction equation, representing the demand for investment activity:

$$
B_{t}=B^{0}+B^{1} t+\beta Z_{t}+\beta_{1} Z_{t-1}
$$

3 . A reaction equation, indicating the spending of nonlabour income:

$$
F_{t}^{\prime}=\epsilon Z_{t}+\epsilon_{1} Z_{t-1}+C^{0}+C^{1} t .
$$

1 In a monograph to be published by Messrs. Hermann and Cie (Paris). Some of the figures have been improved since. For typographical reasons the notation has been changed. Capital letters in this paper correspond to double-barred variables in my other publications. 
TABLE 1

Variables Used in the Simplified Model for the United States, 1919-1932

\begin{tabular}{|c|c|c|c|c|c|c|c|c|c|c|c|c|c|c|c|c|}
\hline ort description) & Symbol & Unit & 1919 & 1920 & 1921 & 1922 & 21923 & 31924 & 1925 & 1926 & $\mid 1927$ & 1928 & 1929 & 1930 & 1931 & 1932 \\
\hline 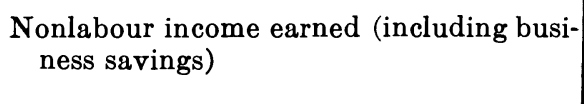 & & $\begin{array}{r}\text { Aver } \\
1919 \\
=1\end{array}$ & 107 & 98 & 70 & 89 & 101 & 105 & 124 & 119 & 122 & 138 & 132 & 90 & 62 & 42 \\
\hline Tonworkers' consumption outlay & $B_{t}^{* *}$ & $\begin{array}{l}\text { a } \\
\text { b }\end{array}$ & $\begin{array}{l}23 \\
80\end{array}$ & $\begin{array}{l}25 \\
71\end{array}$ & $\begin{array}{l}16 \\
68\end{array}$ & $\begin{array}{l}20 \\
76\end{array}$ & $\begin{array}{l}24 \\
72\end{array}$ & $\begin{array}{l}21 \\
77\end{array}$ & $\begin{array}{l}22 \\
83\end{array}$ & $\begin{array}{l}24 \\
92\end{array}$ & $\begin{array}{l}21 \\
90\end{array}$ & $\begin{array}{l}21 \\
94\end{array}$ & $\begin{array}{l}23 \\
98\end{array}$ & $\begin{array}{l}18 \\
81\end{array}$ & $\begin{array}{l}13 \\
70\end{array}$ & $\begin{array}{l}10 \\
68\end{array}$ \\
\hline
\end{tabular}

- Unit such as to make total work done in all industries equal to 100 in the average for 1919-1932.

b With my figures this unit makes the average value of $Z$ over 1919-1932 equal to 100 ; therefore the unit for $Z$ is also $\$ 34.4 \times 10^{\circ}$ and equals that for $F$.

Computation of these values.

$Z_{\text {t }}$ Based upon data given in America's Capacity to Consume (Moulton, Leven, Warburton, et al.) for the years up to 1929 and upon the official estimates of the Department of Commerce (Kuznets). The figures include not only dividends, interest, net royalties and rents, and entrepreneurial withdrawals, but also one-half of the salaries and, finally, estimates for speculation gains, as given in the above work.

$B$. Employment figures for the metal industries multiplied by such a factor as to make the average of total employment in industry equal to 100 for the period 1919-1932. It is assumed that the average level of employment in investment industries covers the same proportion of total employment as the proportion between gross capital formation (taken from Kuznets, Bulletin of the National Bureau of Economic Research, No. 52) and national income.

$F$, Found by subtracting the total wages bill (i.e., in the terminology of the above-cited sources on national income, wages plus onehalf of the salaries) from the total value of consumers' goods. The latter item has been taken for 1919, 1921, 1923, 1925, 1927, and 1929 from Warburton's estimates ("Value of the Gross National Product and its Components, 1919-1929," Journal of the American Statistical Association, Vol. 29, Dec., 1934, pp. 383-388), and for the other years found by interpolation and extrapolation with the aid of F.R.B. indices, agricultural, automobile, mineral, and railway statistics.

It should be borne in mind that the relevant features in our figures are only their movements around their trends and that these movements are only slightly changed if some alternative methods of computation are used. 
The justification for this special choice of the form of our equations has been given elsewhere. ${ }^{2}$

The quantities $A^{0}, A^{1}, B^{0}, B^{1}, C^{0}, C^{1}, \zeta_{B}, \zeta_{z}, \beta$, and $\epsilon$ are supposed to be changing only slowly and are treated as constants.

\section{STATISTICAL DETERMINATION OF EQUATIONS}

Two methods are possible, in principle at least, which may be called the structural and the historical method.

The structural method tries to measure immediately the constants appearing in the equations or to deduce them by reasoning in connection with direct measurement. In order to do so, one must be clear about the meaning of the coefficients. An example will be given while discussing the American model in this respect.

The historical method is that usually known as the method of correlation analysis. The chief difficulties are:

1. Theoretical:

(a) The possibility that disturbances do not follow a simple statistical law of distribution;

(b) The possibility of multicollinearity;

2. Practical:

(a) The availability of no data or only bad data;

(b) The availability of only annual figures, making difficult the exact determination of lags. ${ }^{3}$

The problems met in calculating the coefficients of equations (1) to (3) are in many respects illustrative of these difficulties. Some details may be given.

Equation (1): This equation contains, strictly speaking, not only the coefficients $A^{0}, A^{1}, \zeta_{B}$, and $\zeta_{Z}$ as unknown coefficients, but also the time structure of the corresponding terms, i.e., the lag of the term $\zeta_{B} B_{t-1}$ and the two lags included in the term $\zeta_{Z}\left(Z_{t-1}-Z_{t-2}\right)$. Now it is scarcely possible to determine these time structures really exactly with the help of only annual data, which we may indicate by

and

$$
Z_{t}^{* *}=Z_{t-1}+Z_{t}+Z_{t+1} \text { for the year centered at time } t,
$$

$$
Z_{t-3}{ }^{* *}=Z_{t-4}+Z_{t-3}+Z_{t-2} \text { for the preceding one, etc. }
$$

For that reason they have been assumed a priori to be as they are written down. The considerations upon which the choice is based are:

${ }^{2}$ Cf. (a) Revue Trim. de l'Institut Int. de Stat., July, 1936; (b) Weltw. Archiv, Vol. 45, 1937, p. 42; (c) Archiv für math. Wirtschafts- und Sozialforschung, Vol. 3,1937, p. 7.

${ }^{3}$ In fact only annual figures are available for the two variables $Z$ and $F^{\prime}$ which are of vital importance to our analysis. 
I. The meaning of the lag in $\zeta_{B} B_{t-1}$ is that it equals about one-half of the period of production in investment-goods industries; a period of production of 8 months seems to be a fair assumption, and therefore $\zeta_{B} B_{t-1}$ was supposed to lag 4 months. Furthermore, $B_{t-1}$ has been approached by the formula

$$
3 B_{t-1}=2 B_{1}+B_{t-3}=\frac{2}{3} B_{t}^{* *}+\frac{1}{3} B_{t-3}^{* *},
$$

which holds exactly only in the case of rectilinear development of $B$, but approximately also for the cases in which we are interested. The right-hand member of (I) has, therefore, been introduced as one variable in our calculations.

II. Statistically speaking, the only practical possibility to represent speculative gains with the help of only annual figures is to take consecutive differences. In our notation, this would be $\zeta_{Z}\left(Z_{t}{ }^{* *}-Z_{t-3}{ }^{* *}\right)$ $=\zeta_{Z}\left[\left(Z_{t+1}+Z_{t}+Z_{t-1}\right)-\left(Z_{t-2}+Z_{t-3}+Z_{t-4}\right)\right]$. Now if $Z$ shows a movement that for intervals of two years may be approximated by a straight line, this expression may be simplified in various ways. In our example we choose the one leading to the simplest formulae, viz.:

$$
\zeta_{Z}\left(Z_{t}^{* *}+Z_{t-3}^{* *}\right)=9 \zeta_{Z}\left(Z_{t-1}-Z_{t-2}\right) \text {. }
$$

As the hypothesis of a rectilinear development of $Z_{t}$ is only a very rough approximation, it would be interesting to make a refined investigation with perhaps interpolated values for $Z$. As my purpose is, however, only to give an example, I do not go into this question now. ${ }^{4}$

With this given time structure, the coefficients $A^{0}, A^{1}, \zeta_{B}$, and $\zeta_{Z}$ have been determined by the historical method applied to the period 1920-1931. ${ }^{5}$ Using the elementary regression on $Z_{t}^{* *}$ one finds:

$$
\begin{aligned}
Z_{t}{ }^{* *}=\left(F_{t}{ }^{\prime}\right. & \left.+B_{t}\right)^{* *}+2.1 B_{t}^{* *}+1.05 B_{t-3}{ }^{* *} \\
& +0.36\left(Z_{t}{ }^{* *}-Z_{t-3}{ }^{* *}\right)+0.81 t^{* *}-66 .
\end{aligned}
$$

Here, as is indicated by the double asterisks, all figures are annual totals, as given in the tables. The symbol $t^{* *}$ indicates time in half years elapsed since the middle of the period taken (i.e., Dec. 31, 1926). Writing for the double asterisk symbols their expressions and using (I) and (II), the above equation can be transformed into one for "four-month variables." At the same time $t^{* *}$ should be replaced by $t$ (whose units are 4 months).

We obtain:

$$
Z_{t}=F_{t}{ }^{\prime}+B_{t}+3.15 B_{t-1}+1.08\left(Z_{t-1}-Z_{t-2}\right)-22+0.18 t
$$

4 Also in some cases of nonrectilinear development the approximation chosen would be valid.

5 The year 1932 has not been used, because afterwards the impression was obtained that a strong, more or less extra-economic, shock was produced by the fall of sterling. 
The coefficient $\zeta_{B}$ may be checked by the structural method. A closer analysis of the meaning of the term $\zeta_{B} B_{t-1}$ tells us that it is generated from the expression, "value of means of production produced minus wages paid in corresponding industries," from which it differs by a con-

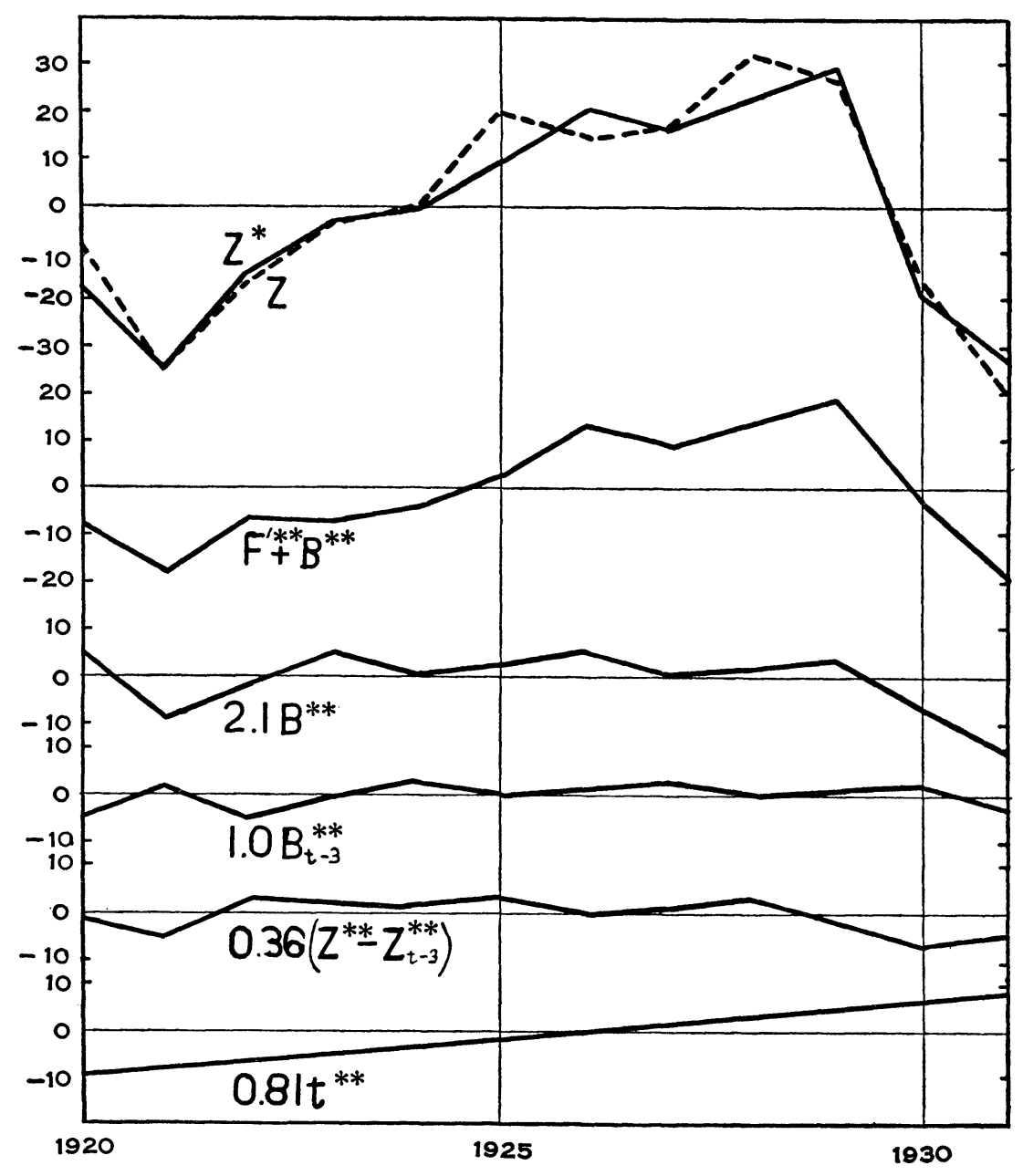

Figure 1.-Explanation of movements in $Z$ by formula (1).

stant plus a trend. Writing $Q_{t}$ for the prices of means of production and $Y_{t}$ for their production, then the part of $Z_{t}$ corresponding to investment industries should be:

$$
Q_{t-1} Y_{t-1}-L_{t-1} B_{t-1} \text {. }
$$

In this expression, $Q$ and $Y$ move about parallel to $B$; the relation between $Q$ and $B$ depending on the changes in productivity, and that 


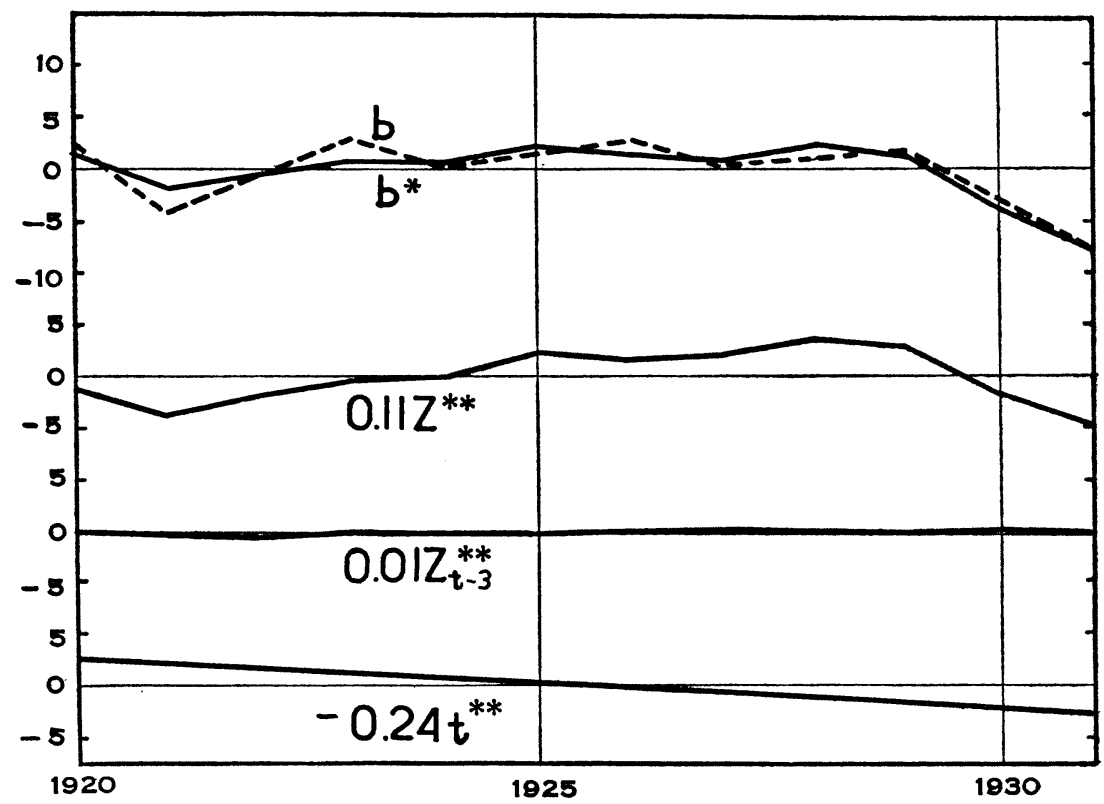

Figure 2.-Explanation of movements in $b$ by Formula (2).

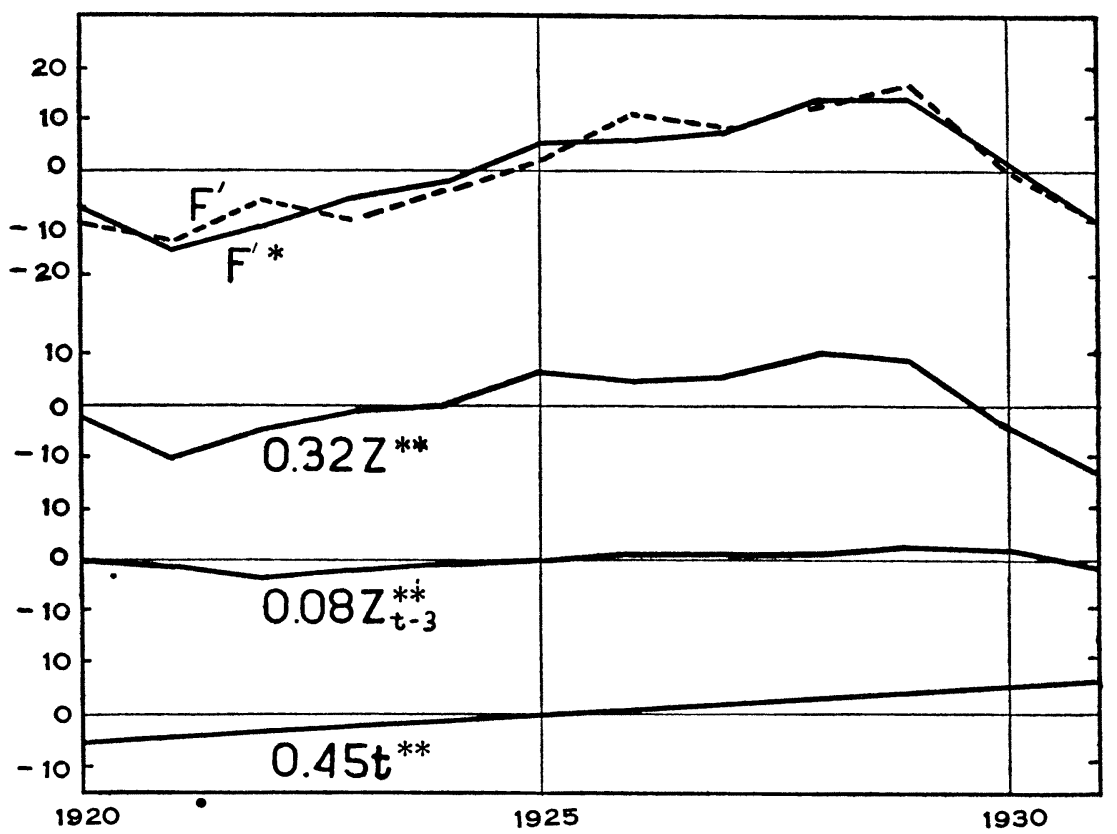

Frgure 3.-Explanation of movements in $F^{\prime}$ by formula (3). 
between $Y$ and $B$ depending, in addition, on the slope of the supply curve. A rough check shows that the coefficient found is realistic.

Equations 2 and 3: To these equations the historical method has been applied in such a form that a regression equation has been established with the values for $Z$ in the same year and the year before and with time as independent variables. This equation has been reduced to an equation in $Z_{t}$ and $Z_{t-1}$ (i.e., the values for $t$ and for 4 months before) with the help of the relation, similar to (I):

$$
3 Z_{t-1}=\frac{2}{3} Z_{t}^{* *}+\frac{1}{3} Z_{t-3}^{* *}
$$

in the notation just introduced. Using elementary regressions, we find

$$
B_{t}=0.09 Z_{t}+0.03 Z_{t-1}+2.3-0.05 t
$$

and

$$
F_{t}^{\prime}=0.16 Z_{t}+0.24 Z_{t-1}+0.10 t
$$

Figures 1, 2, and 3 show for each of the three variables: (i) the observed values (deviations from average), dotted line; (ii) the calculated values (with asterisk), first full line; and (iii) the components of the calculated values. The latter are indicated by their mathematical expressions, which are given in terms of the annual figures used in the correlation analysis and given in the table. For that reason the coefficients are not those of (4), (5), and (6); these latter would be obtained by applying (I), (II), and (III).

\section{EQUILIBRIA AND EQUILIBRIUM DEVELOPMENTS}

A first step to find the properties of a given economic system, represented by its system of dynamic equations, is the determination of its equilibria and its equilibrium developments. By an equilibrium I understand a set of time-constant values of variables satisfying the equations. If such a set exists, the economic system possesses a stationary state. In general this will not be the case, but there will exist, among the possible movements of the system, movements that are distinguished by a more continuous, a more smooth development than other possible movements. I have called them "equilibrium developments" and shown that an economic system represented by a linear system of equations with only linear functions of time as "known terms" is able to carry out a linear movement in time. Systems with more complicated "known terms" will, in general, show more complicated "simplest movements," which, in their case, could be taken as equilibrium developments. But anyhow such an equilibrium development can be found ${ }^{6}$ and, in the case of linear equations with arbitrary

${ }^{B}$ Cf. my paper, "Einige Grundfragen der mathematischen Konjunkturtheorie," Archiv für mathematische Wirtschafts- und Sozialforschung, Vol. 3, 1937, p. 1. 
"known terms," it is always possible to obtain homogeneous equations (i.e., equations without "known terms"). This can be done by measuring all variables by their deviations from the equilibrium development instead of by their absolute ("natural") values. These deviations would be comparable to trend deviations in the statistical language. The movements of these deviations, which are in fact the movements in which business-cycle research is most interested, would thus be governed by simpler equations easier to handle mathematically.

In our example this would lead us to the following set of equations for the deviations from the equilibrium development:

$$
\begin{aligned}
z_{t} & =f_{t}{ }^{\prime}+b_{t}+3.15 b_{t-1}+1.08\left(z_{t-1}-z_{t-2}\right), \\
b_{t} & =0.09 z_{t}+0.03 z_{t-1}, \\
f_{t} & =0.16 z_{t}+0.24 z_{t-1} .
\end{aligned}
$$

In these equations the lower-case symbols are the deviations corresponding to the capital ones used previously.

The equilibrium position or development may be stable or unstable; this depends on the nature of the small movements generated by some small initial deviation from equilibrium. Stable equilibrium will be present if these movements tend to approach, in the long run, the equilibrium.

\section{NECESSITY OF SIMPLIFICATIONS}

In at least three respects the business-cycle mechanism is complicated: (1) as to the economic structure (the number of variables and relations is large); (2) as to the time structure (the number of different lags and lag distributions is large); (3) as to the mathematical structure (complicated functions play a role). In order to be workable at all, the model must be simplified. Many interesting features of business-cycle problems can only be seen after the solution of the equations; and in order to be solved these must be workable.

Simplifications in economic structure. Two types of simplifications may be mentioned in this section, viz., "macro-analysis" and "incidental simplifications." By "macro-analysis" (Frisch) is meant the grouping of a number of variables of the same type as index numbers or totals (price indices, value totals, etc.). It is common to almost all business-cycle theories and could scarcely be dispensed with. How far it is legitimate is, however, not easy to see. This will, nevertheless, not be discussed here; it seems to me that only concrete figures can lead here to fruitf ul statements.

"Incidental simplifications" are present as soon as in a certain equation some terms are neglected; this will in general be justified by the smallness of these terms. A general case is the neglecting of all influences that are almost constant or trend influences. For this reason, the 
demand for replacement of capital goods may be neglected, as far as its deviations from trend are concerned.

Another example of an important "incidental simplification" is the neglecting of the influence of interest rates (in the narrower sense of short or long market rates for lending) on investment, which, it seems to me, is in many cases justified. ${ }^{7}$

An interesting type of simplification has been suggested by $\mathrm{Mr}$. Haavelmo at the Oxford meeting of the Econometric Society, 1936. It might be formulated as follows. Suppose we have a system with $n$ variables. Suppose further that we are only interested in the movements of a small number $m$ of these variables, relating to one special field or market and that it is possible to eliminate a number of the variables in which we are not interested. We may then be left with one or more equations between the noninteresting variables, which no longer represent direct causal relationships but only indirect ones, as they are elimination results. The coefficients of such equations and even their mathematical form may then be obtained immediately from observation, instead of being found by elimination calculations with elementary coefficients derived themselves from observation. In other words, these equations may immediately be deduced from observations. They are, however, no longer interpretable; they are perhaps numerically more exact and easier to handle in further computations.

\section{SIMPLIFICATIONS IN TIME STRUCTURE}

Simplifications in time structure may be, above all, simplifications in the lags occurring in the system. In principle, a considerable number of the most different lags will exist, which are even incommensurable. An important step towards simpler formulas is their replacement by commensurable ones. In addition, their number may be reduced by neglecting small ones or by reducing "distributed lags" (Irving Fisher) to simpler ones. An example of the latter will be given.

It will be noted that our illustrative system shows a very much simplified time structure. I think this is in so far legitimate, as static theory did not consider any time structure at all, and we have, therefore, still to learn the very simplest consequences of the introduction of time structures. These very much simplified time structures may, however, lead into oversimplifications where even the relevant features are eliminated. A clear example can be quoted. Suppose that we simplified our model to such an extent as to be left with only two different time points in the final equation. This could always be attempted by taking as the time unit the largest lag appearing in that equation and by replacing a lag of intermediate length by a combination

${ }^{7}$ I hope to have an opportunity to go into this problem more at length. 
of a zero lag and a unit lag. In concreto: if two years were the largest lag appearing in a final equation

$$
x_{t}+a_{1} x_{t-1}+a_{2} x_{t-2}=0 \text { (time unit } 1 \text { year), }
$$

we could write for $a_{1} x_{t-2}: \frac{1}{2} a_{1} x_{t}+\frac{1}{2} a_{1} x_{t-2}$, which under certain conditions is not so bad an approximation, and which would change our final equation into:

$$
\left(1+\frac{1}{2} a_{1}\right) x_{t}+\left(a_{2}+\frac{1}{2} a_{1}\right) x_{t-2}=0 \text { (time unit } 1 \text { year), }
$$

or

$$
\left(1+\frac{1}{2} a_{1}\right) x_{t}+\left(a_{2}+\frac{1}{2} a_{1}\right) x_{t-1}=0 \text { (time unit } 2 \text { years). }
$$

This would, however, be simplification of a dangerous nature, as such an equation can no longer show cycles of any length, but only very short ones, or no waves at all. ${ }^{8}$

Of course, every reduction in the number of terms of the final equation means a certain "impoverishment" of the picture. A four-term equation may show two superposed cycles, whereas a three-term one can only show one cyclic component, etc. But it seems that, in particular, the reduction of a three-term equation to a two-term one is a heavy impoverishment, as the movements corresponding to a threeterm equation still may show any period, whereas those of a two-term one can only be either very short-period or nonperiodic movements.*

\section{SIMPLIFICATIONS IN MATHEMATICAL FORM OF RELATIONSHIPS}

A third type of simplifications is obtained by the introduction of simpler types of functions instead of the functions that in principle should have been chosen. One very general procedure is the approximation by linear functions of all relations entering into the system. This is a powerful help for further calculations, as linear equations are in many cases solvable explicitly, which is practically never so for nonlinear equations. ${ }^{9}$

For this reason I prefer linear formulas for demand functions, etc.

${ }^{8}$ Even in the latter case, however, the first extrapolations obtained by such an equation may have a certain value. Cf. Section XI below.

* Even such a system, whose characteristic equation gives only one component, namely an exponential (apart from trivial cycles shorter than the distance between observations), will, however, produce real observable cycles when it is exposed to erratic shocks. These cycles will be "changing harmonics" and their average frequencies can be determined from the coefficients of the characteristic equation. Investigations which $\mathrm{Mr}$. Haavelmo and I have quite recently undertaken on American data have led us to suspect that many structures in economic reality have, from the viewpoint of the characteristic equation, only an exponential component.-EDitor's Footnote.

${ }^{9}$ A closer examination of the implications of such a type of simplification was considered in my paper: "Utber den Wert mathematischer Konjunkturtheorien," Beiträge zur Konjunkturlehre, Hamburg, 1936. 
instead of constant-elasticity formulas in cases where the statistical material is not strongly against this hypothesis. When the linear hypothesis is adopted it is not important whether the elasticity of a certain demand or supply function is different in boom and depression periods, but it is important whether its slope is different. ${ }^{10}$

The linear approach is, however, too rough in some cases of special importance to business-cycle theory. A good example is that of the phenomenon of saturation, which, for example, may show itself in the following case: Suppose full employment is reached without a turning point occurring. The boom may then still continue and show itself in higher wages, prices, etc. Evidently, however, the relationship determining the volume of employment must show curvilinearity in some respect. To take the case of our equation (8), $b_{t}$ cannot continue to be the same linear function of $z_{t-1}$, after the state of full employment, say $b_{f}$, has been reached. It will, in fact, be a relation showing an asymptote for $b_{t}=b_{f}$, and an extrapolation of our equation (2) for $b_{t}>b_{f}$ will not be admissible. As, however, it would be rather difficult to work with non-linear equations, one might here use the method of approximating the asymptotic function by two functions. Of these, the first would be equation (8), valid only for $b_{t}<b_{f}$, whereas the second would be $b_{t}=b_{f}$, valid only if $z_{t-1} \geqq b_{f} / \beta$. Probably other equations would have to be changed at the same time, e.g., the equation determining $q_{t}$; because one has to expect that the impossibility of a further increase in production, aside from a still growing demand for investment goods, will raise prices, although $b_{t}$ remains constant.

The discussion of the system's movements must then be split up into two parts; first, a discussion for $b_{t}<b_{f}$, and secondly, one for $b_{t}=b_{f}$. The systems of equations would be, e.g.:

$$
\begin{array}{rlrl}
\text { Case I: } b_{t}<b_{f} & & \text { Case II }: b_{t}=b_{f} \\
z & =f^{\prime}+2 b_{-1}+0.4 q+\zeta_{z}\left(z_{-1}-z_{-2}\right) & & z=f^{\prime}+2 b_{-1}+0.4 q+\zeta_{z}\left(z_{1}-z_{-2}\right) \\
b & =\beta z_{-1} & & b=b_{f} \\
f^{\prime} & =\epsilon_{1} z+\epsilon_{2} z_{-1} & f^{\prime} & =\epsilon_{1} z+\epsilon_{2} z_{-1} \\
q & =k b_{-1} & & q=k^{\prime} z_{-1}
\end{array}
$$

The first and third equations would have remained the same; but the second and fourth have changed. Whether the fourth equation would become $q=k^{\prime} z_{-1}$ or perhaps $q=k^{\prime} z_{-2}$, or still another one, would have to be seen from observations. The chief remark to be made now is that the character of the movements may have been changed. It might be, e.g., that in Case II a shorter period is shown than in Case I. It might even be that Case I does not show a periodic movement at

10 Haberler, Depression and Prosperity, Geneva, 1937, attaches a great importance to changes in elasticities for the explanation of the "turning points." 
all, whereas Case II might do so. In that case, Haberler's distinction between the analysis of the cumulative movement and the turningpoint analysis would be translated in a simple way into mathematical formulae. ${ }^{11}$ The two cases would at the same time correspond to the "first" and the "second" stage of the boom ("erster und zweiter Anstieg" of Spiethoff). It must be added that for the United States between 1919 and 1932 a state of full employment in the investment industries with a corresponding overnormal rise in prices has hardly existed and that in that case our former analysis seems to apply. The history of German business cycles before the war seems, however, to show interesting examples of the second stage of the boom.

There are still other forms of simplifications. They were already indicated in my "Survey" and the reader may be referred to that paper. ${ }^{12}$

\section{CHARACTER OF MOVEMENTS}

The character of the system's movements can be determined most easily and explicitly in the following special case: (a) it is possible to find one "final equation" by an elimination of all variables but one and this equation is linear. It is easy to see then that two sorts of components are, in general, possible: cyclical and noncyclical; the latter either tending to an equilibrium ${ }^{13}$ or moving away from an equilibrium. Although this case is a special one, many more general cases can, with a certain degree of approximation, be reduced to it.

In the case of a nonlinear final equation there exist other equilibrium positions or developments than the one chosen as reference development and much depends on the situation of these other equilibria, which may be stable or unstable. These can be found by supposing the deviations from trend of the variable left as constants and solving the final equation. If there are several such equilibrium situations within the field of economic relevant values, the movements may be far more complicated. ${ }^{14}$

In our simple example we can easily obtain one final equation by eliminating $b_{t}$ and $f_{t}{ }^{\prime}$; we are left with an equation in $z_{t}$ :

$$
\begin{aligned}
0.75 z_{t}-1.63 z_{t-1}+0.99 z_{t-2} & =0 \text { or } \\
z_{t}-2.18 z_{t-1}+1.32 z_{t-2} & =0 .
\end{aligned}
$$

${ }^{11}$ Haberler, op. cit.

12 "Annual Survey: Suggestions on Quantitative Business Cycle Theory," Econometrica, Vol. 3, July, 1935, pp. 241-308.

${ }^{13}$ This term being understood now to include also "equilibrium developments" (cf. section IV).

${ }^{14}$ I treated (constructed) examples in the following papers: "Über den Wert mathematischer Konjunk turtheorien," loc. cit., and "Ưber die Sekundarwirkungen zusätzlicher Investitionen," Weltwirtsch. Archiv, January, 1937, p. 39. 
The characteristic equation corresponding to this difference equation is

$$
x^{2}-2.18 x+1.32=0,
$$

with the roots

$$
\begin{aligned}
& x_{1}=1.09+0.36 i \quad(i=\sqrt{-1}), \\
& x_{2}=1.09-0.36 i .
\end{aligned}
$$

The general solution of (10) is, therefore,

$$
z_{t}=c \sqrt{1.32} t \sin (19.6 t+d)
$$

representing a cyclic movement with a period of some 6 to 7 years, with rather rapidly increasing amplitude.

\section{VARIATION PROBLEMS}

These are the problems of the influence of a change in constants on the movements. The setting of these problems is in itself often difficult. Formulated rather generally, the difficulty is that a change in one constant often entails a change in another and that to assume only the first constant changed would be economic nonsense. A circumstance of special importance belonging to this type of difficulty is that the movements of a system are not determined by the coefficients of the equations only but also by the initial values of the variables. Therefore it must be known whether an assumed change in some coefficient entails a change in initial values. Examples may illustrate the difficulty.

Compensating public works. The shifting of public works from prosperity to depression may be considered as a case of changing coefficients, viz., reducing the $\beta$ 's, say, to one-third of their former values. Instead of our final equation (10) we now get

or

$$
0.81 z-1.42 z_{-1}+1.05 z_{-2}=0
$$

$$
z-1.75 z_{-1}+1.30 z_{-2}=0 .
$$

The roots of the characteristic equation

$$
x^{2}-1.75 x+1.30=0
$$

are

$$
x_{1,2}=0.87 \pm 0.74 i
$$

showing that the movements are slightly less explosive but have a considerably shorter period, viz., of about 3 years.

A question of large practical importance, viz., whether the amplitude of the cycles will be diminished by such a policy cannot, however, be answered yet by this statement. Amplitudes are largely determined by initial conditions; only relative amplitudes are fixed by the co- 
efficients of the equations. Thus it is quite clear that the proportion of the amplitude of $b$ to that of $z$ is diminished; but the question remains whether the amplitude of $z$ will be larger or smaller. This apparently depends on whether the initial shock leading to the cyclical movements usually appears in $b$, in $z$, in both, or in other variables. A multitude of possibilities presents itself.

(i) Suppose, first, that a system usually is brought out of equilibrium by only one shock in only one variable and that in this way the cycle is generated. If, now, this shock is caused by an important invention, the first deviation from equilibrium will show itself in $b$, representing investments for new industries, not based on previous profits. This initial deviation in $b$ will then be the same in the case without and in that with compensating public works; and the corresponding initial deviation in $z$ will also be the same, as it is found from equation (1). The further development will be different because of a difference in period as well as one in dampening degree. As the period is shorter in the case with compensating public works, the system cannot reach such a large amplitude as it could without compensating public works. $\mathrm{As}_{\mathrm{s}}$, in addition, the dampening degree is somewhat smaller, this conclusion is reinforced. The influence of the policy discussed will, therefore, in this case, be a decidedly favorable one, far more than one would deduce from the (only small) change in the damping degree.

(ii) Suppose now, however, that a change in crops is usually the initiating cause. In our simplified model this would mean that $z$ at that moment shows a value different from the one found by formula (1): the crop change would change nonlabour income for another reason than those represented in that equation. The initial deviation would now occur in $z$ and, therefore, be the same in the case without and in that with compensating public works. Because of equation (2) we should now find a different initial deviation in $b$ in the two cases. It would be smaller in the case with compensating public works. As, in addition, the same arguments of period and dampening changes apply, the amplitude of $b$ will now be reduced still more than formerly by the compensating policy.

(iii) In general, however, a complex of causes, partly from remote times, will be the origin of disequilibrium; and then the problem is somewhat more complicated. In every case, however, the "initial values" influence the result of any form of business-cycle policy. And these initial values are not only an expression for the phase of the cycle in which society finds itself at the moment the policy starts, but also for the "localization" of new disequilibria likely to occur: for the fact whether these disequilibria are more concentrated upon one part or more upon another part of society. 


\section{FURTHER EXAMPLES ON BUSINESS-CYCLE POLICY}

We continue our examples of variation problems by considering the consequences of a reduction in speculation opportunities, leading, say, to a reduction of the realized speculative gains to one-half of their previous value. This would mean, in our mathematical language, that the equation for $z$ is changed into:

$$
z=f^{\prime}+b+3.15 b_{-1}+0.54\left(z_{-1}-z_{-2}\right) .
$$

The other equations would not have changed. In this case the final equation becomes:

$$
z-1.45 z_{-1}+0.60 z_{-2}=0 .
$$

The roots of the characteristic equation are:

$$
x=-0.72 \pm \sqrt{0.52-0.60}=-0.72 \pm 0.28 i
$$

from which we find that the period of the movements is now $5 \frac{2}{3}$ years. And-which is far more important-the movement has become a very much damped one; the amplitude, after about half a period, is reduced to less than 0.13 . The business cycle will, therefore, to a large degree be eliminated by this measure.

Next consider a change in savings habits. Suppose there is more saved in the boom and less in depression, or, which is the same thing, that expenditures are less fluctuating. This would mean, in our language, that the coefficients of the equation for $f^{\prime}$ would become smaller, say $\frac{1}{2}$ of their former value. We get, then, the final equation,

$$
0.83 z-1.51 z_{-1}+0.99 z_{-2}=0 .
$$

This corresponds to a movement with 3.6-year cycles and with a somewhat more stable amplitude (a smaller "explosiveness"). The improvement as compared with the uninfluenced case is not very large. A definitely unfavorable change would, however, take place if savings habits were changing in the opposite direction, viz., if savings were more stable throughout the cycle.

Of course, all that has been said in Section IX about the influence of initial values still applies.

\section{REMARKS AND FIGURES ON THE SIGNIFICANCE OF RESULTS}

I finish this paper with some remarks on the "margin of arbitrariness" in the results.

Beginning with the restricted question of significance in the usual statistical sense, I need not repeat here that there is still much difference of opinion as to what is the best criterion by which to judge the limits of uncertainty in regression coefficients. For instance, instead of the elementary regressions given above, others may be used. A type of 
mean regression I often make use of is that obtained by dividing the elementary regression coefficients by the total correlation coefficient. In the case of two variables only, this type is identical with the diagonal regression. The equations obtained by this method may be caledl "Set 2 " against the original "Set 1." Set 2 then runs:

$$
\begin{aligned}
z & =f^{\prime}+b+3.3 b_{-1}+1.14\left(z_{-1}-z_{-2}\right), \\
b & =0.10 z+0.03 z_{-1}, \\
f^{\prime} & =0.167 z+0.252 z_{-1},
\end{aligned}
$$

leading to the final equation:

$$
z-2.40 z_{-1}+1.42 z_{-2}=0 .
$$

There are, however, a number of other arbitrary assumptions at the basis of our equations.

Consider, for instance, the assumption made as to the lag between $z$ and $b$ (cf. Section III, Subsection I). I shall replace this assumption by another, viz., by one that could be called "the introduction of a free lag." The procedure consists in including in the correlation calculation for $z$ both $b_{t}{ }^{* *}$ and $b_{t-3}{ }^{* *}$ as independent variables and transforming the result in the usual way (cf. Section III) in order to obtain an equation with $b_{t}$ and $b_{t-1}$. This procedure again can be carried out by using an elementary regression equation or by using the sort of mean regression equation just mentioned. The following two alternatives are obtained:

$$
\begin{aligned}
& z=f^{\prime}+1.9 b_{-1}+3.0 b_{-2}+1.47\left(z_{-1}-z_{-2}\right), \\
& z=f^{\prime}+1.9 b_{-1}+3.1 b_{-2}+1.50\left(z_{-1}-z_{-2}\right),
\end{aligned}
$$

to be used, respectively, in "Set 3 " and "Set 4. ."

All the calculations about the movements of the system and the influence of given types of business-cycle policy can now be made with the four sets.

(i) Starting with the calculation of the period of the undisturbed system we find that "Set 2" shows a noncyclic movement, the roots of the characteristic equation being

$$
x=1.20 \pm 0.14 \text {. }
$$

For our purpose-discussion of policy - this is not so serious. What matters is extrapolation for the first few time units (of four months).

(ii) An extrapolation for the first two or three time units gives quite analogous results for the four sets, if we start with a "shock" in a given direction, e.g., $z_{-2}=0 ; z_{-1}=+1$. We find:

$\begin{array}{rrrr}\text { Set 1: } & z_{0}=2.18 & z_{1}=3.43 & z_{2}=4.5 \\ \text { Set 2: } & 2.40 & 4.34 & 7.0 \\ \text { Set 3: } & 2.13 & 3.39 & 4.8 \\ \text { Set 4: } & 2.21 & 3.75 & 5.8\end{array}$


All sets show a "self-reinforcing, cumulative process." The same would, of course, be the case, had a downward "shock" starting from $Z_{-2}=0$ been chosen.

(iii) The results are, however, less homogeneous if we start with an almost horizontal movement, e.g., $z_{-2}=1, z_{-1}=1$.1. We find:

$\begin{array}{lrr}\text { Set 1: } & z_{0}=1.08 & z_{1}=0.90 \\ \text { Set 2: } & 1.22 & 1.37 \\ \text { Set 3: } & 1.20 & 1.31 \\ \text { Set } 4: & 1.31 & 1.67\end{array}$

Again, the divergencies in the levels are not very large, which fact for practical uses may still be of importance. ${ }^{15}$

For the three problems of business-cycle policy we considered above, the following results are found:

Compensating public works
1. $x^{2}-1.75 x+1.30=0$
Period: 36 months
1. $x^{2}-1.89 x+1.39=0$
$39 \mathrm{~m}$.
3. $x^{2}-2.07 x+1.55=0$
$43 \mathrm{~m}$.
4. $x^{2}-2.14 x+1.59=0$
$45 \mathrm{~m}$.

Restriction of speculation opportunities
1. $x^{2}-1.45 x+0.60=0$
Period: 68 months
2. $x^{2}-1.62 x+0.64=0$
3. $x^{2}-1.25 x+0.26=0$
$48 \mathrm{~m}$.
4. $x^{2}-1.32 x+0.24=0$
Expenditures more stable
1. $x^{2}-1.82 x+1.19=0$
Period: 43 months
2. $x^{2}-1.98 x+1.27=0$
$51 \mathrm{~m}$.
3. $x^{2}-1.82 x+1.04=0$
$53 \mathrm{~m}$.
4. $x^{2}-1.87 x+1.03=0$
$62 \mathrm{~m}$.

Combining the three types of policy
1. $x^{2}-0.85 x+0.57=0$
Period: 26 months
2. $x^{2}-0.91 x+0.61=0$
$26 \mathrm{~m}$.
3. $x^{2}-0.96 x+0.61=0$
$28 \mathrm{~m}$.
4. $x^{2}-0.98 x+0.62=0$
$28 \mathrm{~m}$.

Since, for cyclic movements, the damping per 8 months is given by the last term of the characteristic equation (in the form given above), we see that a favorable influence on damping is exercised by restric-

${ }^{16}$ For a fuller exposé of the question whether a separate explanation of turning points is necessary or not, ef. my article "Einige Grundfragen der mathematischen Konjunkturtheorie," Archiv für mathematische Wirtschafts-und Sozialforschung, Vol. 3, 1937. Heft 2. 
tion of speculation and by stabilizing expenditures, in so far as they lead to cyclic movements. Set 2 and Set 4, however, do not show a cyclic movement in the case of speculation restriction. Therefore,other criteria must be used for those cases. These are in the roots of the characteristic equation. Both roots have a modulus below 1 for Set 2, which also means a damped movement. Only in Set 4 one of the roots is 1.1, indicating the possibility of moderately "explosive" movements; these are, however, still less explosive than the movements possible in the uninfluenced system corresponding to Set 2 .

From these and similar analyses we see that the results of various types of policy are less uncertain than the movements of the uninfluenced systems. The smallest spread is shown, however, just in the case most important in practice, viz., the case of a combined policy in the three directions chosen. In fact, the combination shows a very favorable result for all four sets: strongly damped movements with short periods, not differing very much among themselves.

Thus our conclusions concerning (1) the explanation of a cumulative, self-reinforcing process and (2) the effects of business-cycle policy seem more certain than those concerning (1) the explanation of the turning points and (2) the length of the period. This is a rather happy situation, in so far as in fact the chief purpose of business-cycle policy is to prevent the development of cumulative processes.

More generally, if all our equations are of the shape:

$$
\begin{gathered}
x_{t}=\alpha_{11} x_{t-1}+\alpha_{12} x_{t-2}+\cdots+\beta_{11} y_{t-1}+\beta_{12} y_{t-2}+\cdots \\
\quad+\gamma_{11} z_{t-1}+\gamma_{12} z_{t-2}+\cdots \\
y_{t}=\alpha_{21} x_{t-1}+\alpha_{22} y_{t-2}+\cdots+\beta_{21} y_{t-1}+\beta_{22} y_{t-2}+\cdots \\
\quad+\gamma_{21} z_{t-1}+\gamma_{22} z_{t-2}+\cdots \\
z_{t}=\alpha_{31} x_{t-1}+\alpha_{32} x_{t-2}+\cdots+\beta_{31} y_{t-1}+\beta_{32} y_{t-2}+\cdots \\
\gamma_{31} z_{t-1}+\gamma_{32} z_{t-2}+\cdots
\end{gathered}
$$

it can easily be seen that a reduction of all coefficients in the right-hand members, if only effectuated at a sufficiently large degree, finally must lead to a suppression of any disturbance of equilibrium.

League of Nations

Geneva 
http://www.jstor.org

\title{
LINKED CITATIONS \\ - Page 1 of 1 -
}

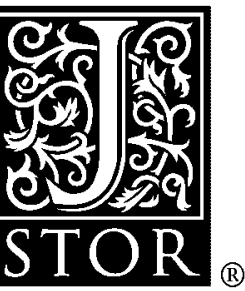

You have printed the following article:

\section{On the Theory of Business-Cycle Control}

\section{J. Tinbergen}

Econometrica, Vol. 6, No. 1. (Jan., 1938), pp. 22-39.

Stable URL:

http://links.jstor.org/sici?sici=0012-9682\%28193801\%296\%3A1\%3C22\%3AOTTOBC\%3E2.0.CO\%3B2-K

This article references the following linked citations. If you are trying to access articles from an off-campus location, you may be required to first logon via your library web site to access JSTOR. Please visit your library's website or contact a librarian to learn about options for remote access to JSTOR.

\section{[Footnotes]}

\author{
${ }^{12}$ Annual Survey: Suggestions on Quantitative Business Cycle Theory \\ J. Tinbergen \\ Econometrica, Vol. 3, No. 3. (Jul., 1935), pp. 241-308. \\ Stable URL: \\ http://links.jstor.org/sici?sici=0012-9682\%28193507\%293\%3A3\%3C241\%3AASSOQB\%3E2.0.CO\%3B2-B
}

NOTE: The reference numbering from the original has been maintained in this citation list. 